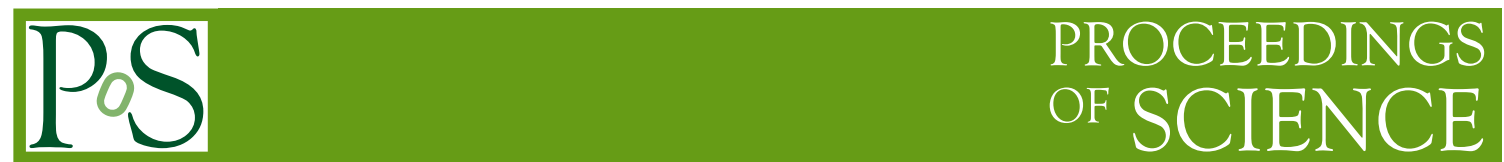

\title{
Two-photon collisions at Belle
}

Hideyuki Nakazawa (Belle Collaboration)

National Central University (R.O.C.)

E-mail: nkzwepost.kek. ip

We report recent measurements of two-photon processes at Belle experiment, cross section measurements for $\gamma \gamma$ to vector meson pair and $\gamma \gamma \rightarrow \eta^{\prime} \pi^{+} \pi^{-}$processes and measurement of $\pi^{0}$ transition form factor in the $\gamma \gamma_{*} \rightarrow \pi^{0}$ process.

36th International Conference on High Energy Physics,

July 4-11, 2012

Melbourne, Australia 

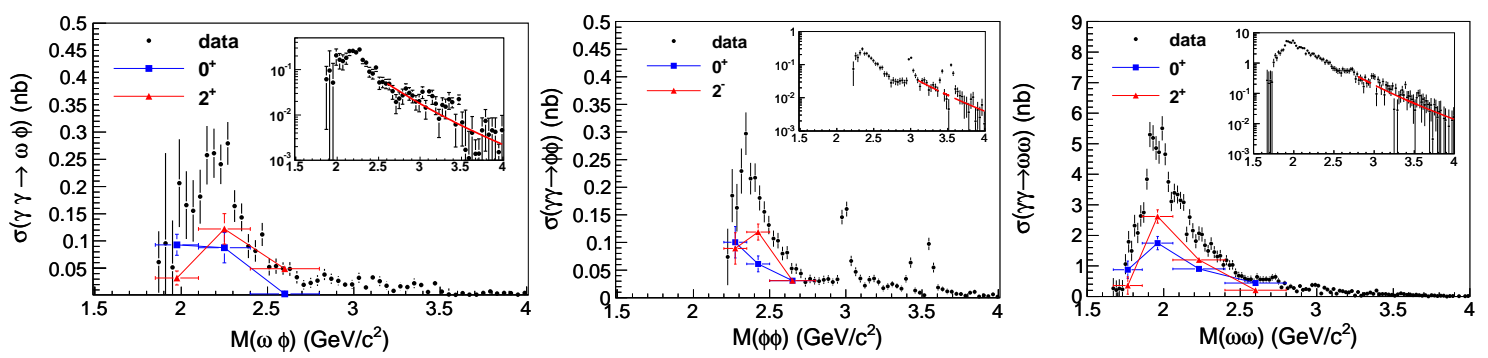

Figure 1: Measured cross sections for $\gamma \gamma \rightarrow \phi \phi, \omega \phi$ and $\omega \omega$ modes. Fit results to $W^{-n}$ dependence are shown in red curves in insets.

\section{1. $\gamma \gamma \rightarrow \phi \phi, \omega \phi$ and $\omega \omega$}

Vector meson pair productions in two-photon process, $\gamma \gamma \rightarrow V V\left(V=\rho, \omega, \phi, K^{*}\right)$ have been measured by TASSO, CELLO and ARGUS experiments (see [四] for review). In most of these measurements, large cross sections are observed not far above threshold. Various models, such as $q^{2} \bar{q}^{2}$ tetraquark states, Regge exchange, an $s$-channel $\rho^{0} \rho^{0}$ resonance, and one-pion-exchange models were proposed to explain the structures.

We measure $\gamma \gamma \rightarrow \phi \phi, \omega \phi$, and $\omega \omega$ modes followed by $\phi \rightarrow K^{+} K^{-}$or $\omega \rightarrow \pi^{+} \pi^{-} \pi^{0}$ decays [[] using an $870 \mathrm{fb}^{-1}$ data sample. Non resonant background events are subtracted by studying $\phi$ - or $\omega$-sideband events. Figure W shows measured cross sections. $\eta_{c}$ (in $\phi \phi$ and $\omega \phi$ modes) and $\chi_{c 0}$ and $\chi_{c 2}$ (in $\phi \phi$ mode) are seen. The products of the two-photon decay width and branching fraction for the charmonia are measured to be $\Gamma_{\gamma \gamma} \mathscr{B}\left(\eta_{c} \rightarrow \phi \phi\right)=7.75 \pm 0.66 \pm 0.62$ $\mathrm{eV}, \Gamma_{\gamma \gamma} \mathscr{B}\left(\chi_{c 0} \rightarrow \phi \phi\right)=1.72 \pm 0.33 \pm 0.14 \mathrm{eV}, \Gamma_{\gamma \gamma} \mathscr{B}\left(\chi_{c 2} \rightarrow \phi \phi\right)=0.62 \pm 0.07 \pm 0.05 \mathrm{eV}$, and $\Gamma_{\gamma \gamma} \mathscr{B}\left(\eta_{c} \rightarrow \omega \omega\right)=8.67 \pm 2.86 \pm 0.96 \mathrm{eV}$.

In all three modes structures are seen near threshold. Their peak position and cross sections measured are summarized in Table $\mathbb{W}$. Any existing models cannot explain observed structures systematically. We perform spin-parity analysis for the structures. Mixture of $0^{+}$(S wave) and $2^{-}$ (P wave) is favored for $\phi \phi$ and $\omega \omega$, and mixture of $0^{+}$(S wave) and $2^{+}$(S wave) for $\omega \phi$.

In higher energy region, $W^{-n}$ dependence of the cross section is measured where $W$ is the invariant mass of the two-photon system. Fit results are shown in insets of Fig. . $n$. $n$ values obtained, $7.2 \pm 0.6$ for $\omega \phi(W>3.2 \mathrm{GeV}), 8.4 \pm 1.1$ for $\phi \phi(W>3.1 \mathrm{GeV})$, and $9.1 \pm 0.6$ for $\omega \omega(W>$ $2.8 \mathrm{GeV}$ ) are consistent with perturbative and non perturbative QCD predictions.

Table 1: Peak position and cross section for the observed near-threshold structures in $\gamma \gamma \rightarrow V V$ processes.

\begin{tabular}{ccc}
\hline mode & peak $\left[\mathrm{GeV} / c^{2}\right]$ & $\mathrm{nb}$ \\
\hline$\omega \phi$ & $\sim 2.2$ & $0.27 \pm 0.05$ \\
$\phi \phi$ & $\sim 2.35$ & $0.30 \pm 0.04$ \\
$\omega \omega$ & $\sim 1.91$ & $5.30 \pm 0.42$ \\
\hline
\end{tabular}



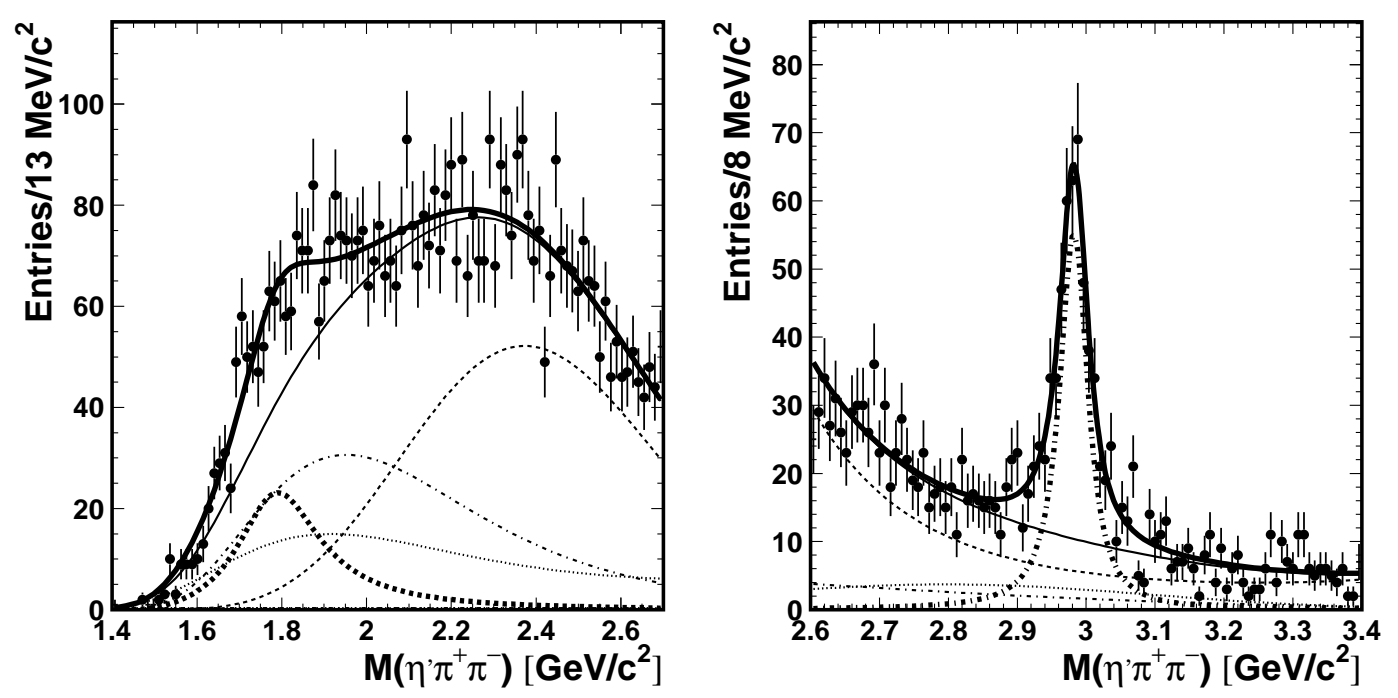

Figure 2: $\gamma \gamma \rightarrow \eta^{\prime} \pi^{+} \pi^{-}$invariant mass distributions in lower energy (left) and $\eta_{c}$ (right) region. Thick solid curve shows best fit, and other curves show resonance, two background, and non resonant components.

2. $\gamma \gamma \rightarrow \eta^{\prime} \pi^{+} \pi^{-}$

The BES II and BES III collaborations observed $X(1835)$ resonance in $\eta^{\prime} \pi^{+} \pi^{-}$invariant mass distribution with more than $20 \sigma$ using $J / \psi \rightarrow \gamma \eta^{\prime} \pi^{+} \pi^{-}$decay mode. Spin-parity of $0^{-+}$is preferred for the resonance. Measured mass and width are not compatible with known resonances. Theoretical consideration on the nature of the $X(1835)$ includes baryonium, glueball and a radial excitation of the $\eta^{\prime}$. The two-photon decay width $\Gamma_{\gamma \gamma}$, which can be measured using two-photon process, is important parameter to study the nature of the resonance.

We reconstruct $\eta^{\prime} \pi^{+} \pi^{-}$in two-photon collisions using $\eta^{\prime} \rightarrow \eta \pi^{+} \pi^{-}$and $\eta \rightarrow \gamma \gamma$ decay modes based on a $673 \mathrm{fb}^{-1}$ data sample [5]. Figure $\square$ shows $\eta^{\prime} \pi^{+} \pi^{-}$invariant mass distributions.

In the lower energy region, an enhanced shoulder is seen around $1.8 \mathrm{GeV}$. We perform an unbinned maximum likelihood fit to the mass distribution taking into account two background, non resonant and peak components. Assuming one peak we obtained $M=\left(1768_{-25}^{+24}\right) \mathrm{MeV} / c^{2}$ and $\Gamma=\left(224_{-56}^{+62}\right) \mathrm{MeV}$ with $4.8 \sigma$ and denote this peak as $\eta(1760)$. If we additionally assume presence of the $X(1835)$ and interference with the $\eta(1760)$, and fix the mass and width of the $X(1835)$ to the values from BES, we found constructive and destructive solutions with the statistical significance of $4.1 \sigma$ for $\eta(1760)$ and $2.9 \sigma$ for $X(1835)$. We also made fits without interference with fixing or floating masses and widths for the resonances. In any fit configurations above, the significance of the $\eta(1760)$ exceeds $4.0 \sigma$. The fit results are summarized in Table $\square$.

For the resonant region the angular distribution of the $\eta^{\prime}$ in the two-photon rest frame is compatible with the assumption that the resonances are pseudoscalars.

A clear $\eta_{c}$ peak is seen (Fig. D). Measured mass and width are consistent with the world average values. The product of two-photon decay width and branching fraction is measured to 
Table 2: Summary of the results for $\eta(1760)$ and $X(1835): M$ and $\Gamma$ are the mass and width; $Y$ is the yield; $\Gamma_{\gamma \gamma} \mathscr{B}$ is the product of the two-photon decay width and branching fraction; $\left(\Gamma_{\gamma \gamma} \mathscr{B}\right)_{90}$ are the upper limits at $90 \%$ confidence level with systematic error included; $\phi$ is the relative phase between the resonances. $S$ is the signal significance including systematic errors.

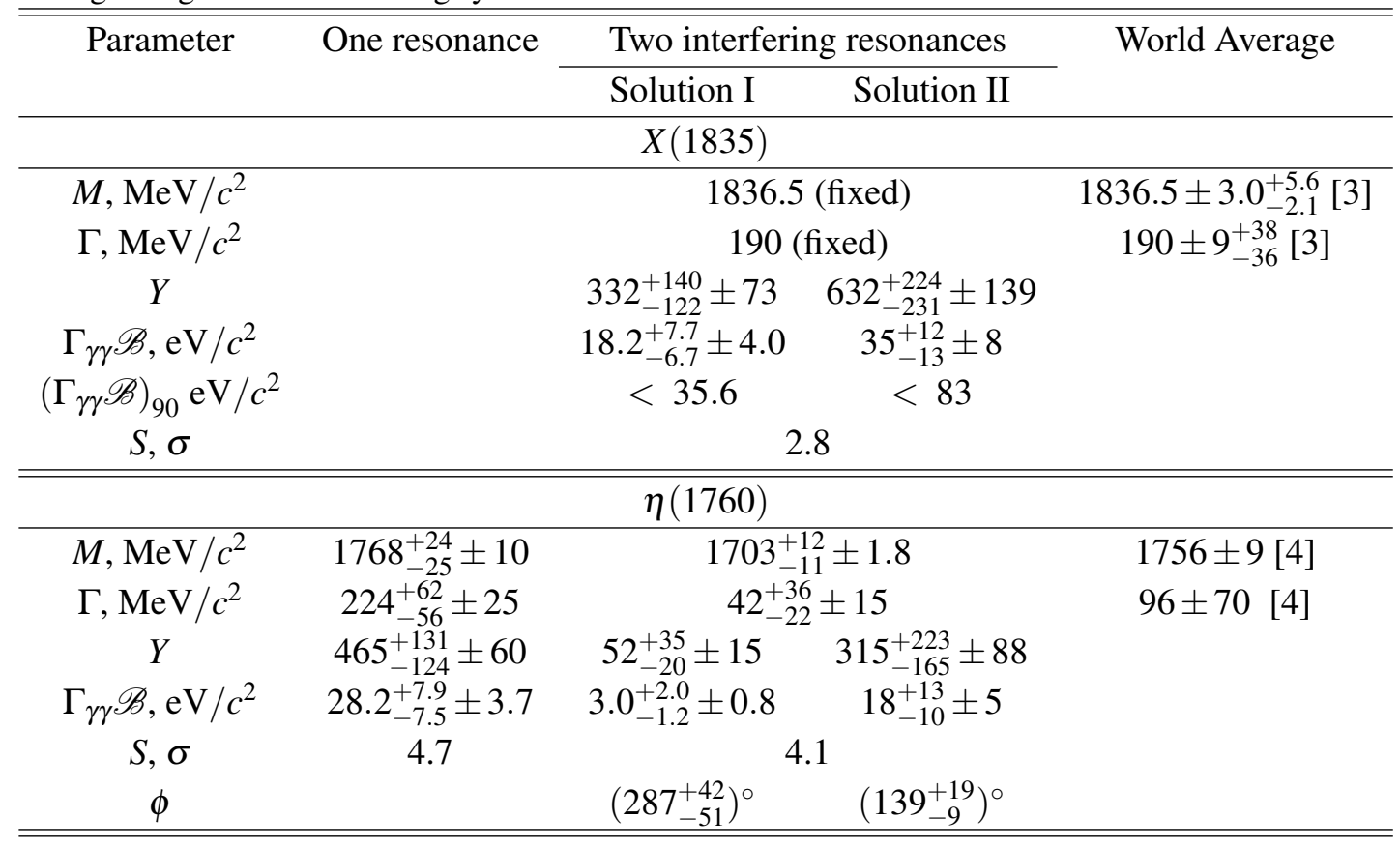

be $\Gamma_{\gamma \gamma} \mathscr{B}\left(\eta_{c} \rightarrow \eta^{\prime} \pi^{+} \pi^{-}\right)=\left(50.5_{-4.1}^{+4.2} \pm 5.6\right) \mathrm{eV} / c^{2}$. In the case the interference effect is included, constructive and destructive solutions are found.

3. $\gamma \gamma * \rightarrow \pi^{0}$

The BABAR experiment measured the $e^{+} e^{-} \rightarrow e^{+} e^{-} \pi^{0}$ process in single-tag mode and obtained differential cross section, $d \sigma / d Q^{2}$, and the $\pi^{0}$ transition form factor (TFF) for $\gamma \gamma * \rightarrow \pi^{0}, F\left(Q^{2}\right)$, with virtuality of the tagged photon, $Q^{2}$, up to $40 \mathrm{GeV}^{2}$ [目]. The result is consistent with previous CELLO and CLEO measurements [ $[\mathrm{D}]$ in $Q^{2}<8 \mathrm{GeV}^{2}$. However in $Q^{2}>10 \mathrm{GeV}^{2}$, the measured transition form factor exceeds asymptotic limit of perturbative QCD by at most $50 \%$. This result has been a hot subject for the last several years, and would imply new physics if confirmed [ 8$]$ ]. $B A B A R$ also reported TFFs for $\eta$ and $\eta^{\prime}[Q]$, and the results are consistent with QCD prediction.

We measure $e^{+} e^{-} \rightarrow e^{+} e^{-} \pi^{0}$ in single-tag mode for $4 \mathrm{GeV}^{2}<Q^{2}<40 \mathrm{GeV}^{2}$ using a 759 $\mathrm{fb}^{-1}$ data sample [ए0]. Signal candidates with one $e^{ \pm}$and two gamma's are selected. $e^{-}$tag (etag) and $e^{+}$tag (p-tag) modes are analyzed separately. We select events in specific angular regions determined with angular correlation between polar angle of $e^{ \pm}$and $\gamma \gamma$ system to avoid harmed region by Bhabha-veto trigger and to ensure sufficient detection efficiency.

$\pi^{0}$ signal yield is obtained by fitting $\gamma \gamma$ mass distribution. Figure B] (left) shows signal yields. Figure [ 3 (right) is the differential cross section $d \sigma / d Q^{2}$ in comparison with results from CLEO and $B A B A R$ measurements, where e-tag and p-tag modes are combined, as we find that the obtained $d \sigma / d Q^{2}$ is consistent between the two modes. 

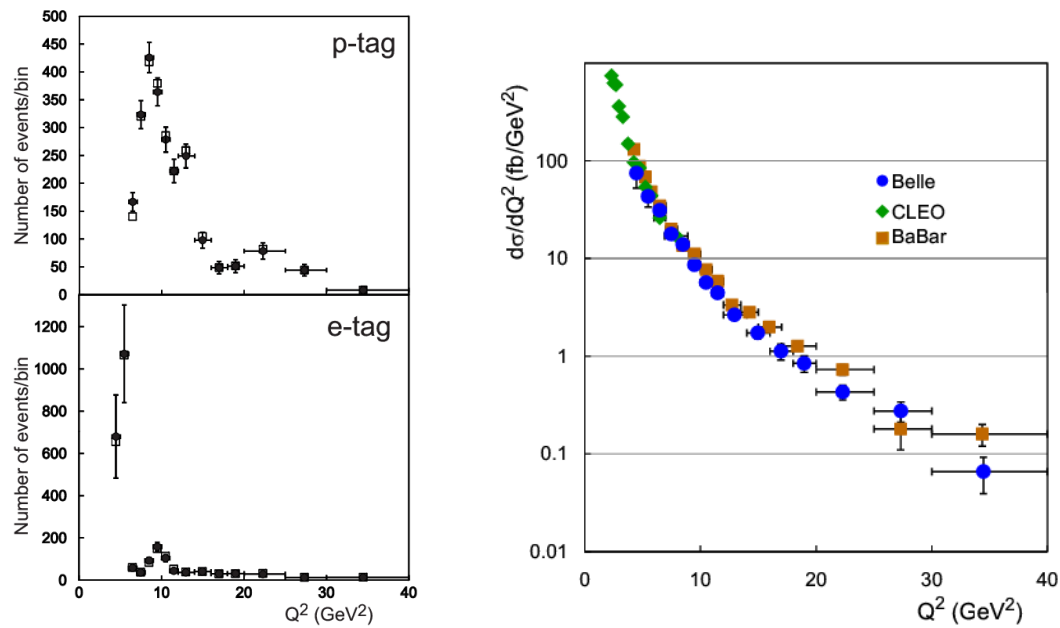

Figure 3: Signal yield (left). Before (open squares) and after (dots with error bars) the $Q^{2}$ unfolding. Differential cross section for $e^{+} e^{-} \rightarrow e^{+} e^{-} \pi^{0}, d \sigma / d Q^{2}$ (right).

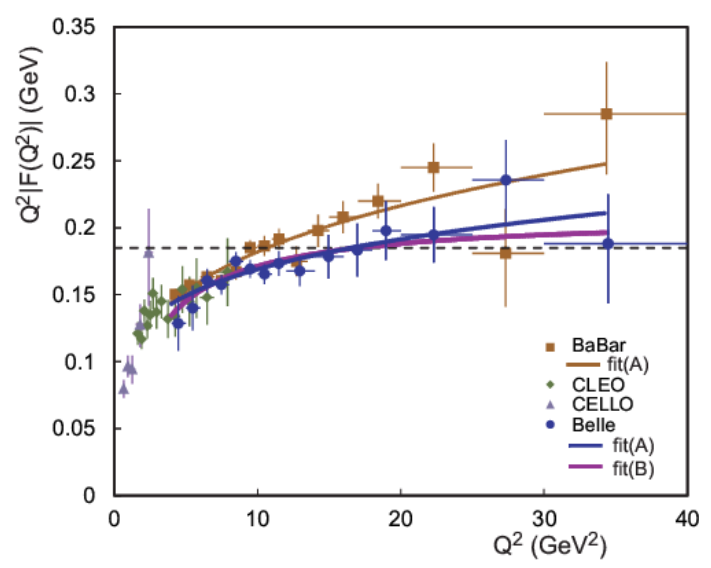

Figure 4: $\pi^{0}$ transition form factor $Q^{2}\left|F\left(Q^{2}\right)\right|$. Curves are fit results. The dotted line shows the asymptotic prediction from $\mathrm{pQCD}$.

The $\pi^{0}$ TFF for the $\gamma \gamma * \rightarrow \pi^{0}$ process is extracted as

$$
Q^{2}\left|F\left(Q^{2}\right)\right|=Q^{2} \sqrt{\frac{d \sigma / d Q^{2}\left(Q^{2}\right)}{2 A\left(Q^{2}\right)}}
$$

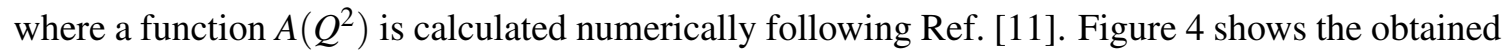
$\pi^{0}$ TFF. In $Q^{2}<9 \mathrm{GeV}^{2}$ region measured results are consistent with each other. However, in the higher $Q^{2}$ region, the Belle results do not show rapid growth observed by $B A B A R$.

We perform fits to $Q^{2}\left|F\left(Q^{2}\right)\right|$ with two different models, one proposed by BABAR [目] (fit(A)) and the other in asymptotic form, $B Q^{2} /\left(Q^{2}+C\right)$ (fit(B)). The fit results are summarized in Table B] and shown in Fig. 田.

In fit(A), the difference of parameter $A$ and $\beta$ between Belle and $B A B A R$ results is about $1.5 \sigma$. We also estimate the difference in $9 \mathrm{GeV}^{2}<Q^{2}<20 \mathrm{GeV}$ region by fitting the data from both experiments together using a function of fit(B), and the difference between deviations of each 
Table 3: Fit results to measured $\pi^{0}$ transition form factor, $Q^{2}\left|F\left(Q^{2}\right)\right|$.

\begin{tabular}{lcll}
\hline & $Q^{2}\left|F\left(Q^{2}\right)\right|$ & \multicolumn{1}{c}{$B A B A R$} & \multicolumn{1}{c}{ Belle } \\
\hline \multirow{2}{*}{ Fit(A) } & $A\left(Q^{2} / 10 \mathrm{GeV}^{2}\right)^{\beta}$ & $A=0.182 \pm 0.005^{\dagger} \mathrm{GeV}$ & $A=0.169 \pm 0.006 \mathrm{GeV}$ \\
& & $\beta=0.25 \pm 0.02$ & $\beta=0.18 \pm 0.05$ \\
\hline \multirow{2}{*}{ Fit(B) } & $B Q^{2} /\left(Q^{2}+C\right)$ & $B=0.209 \pm 0.016 \mathrm{GeV}$ \\
& & $C=2.2 \pm 0.8 \mathrm{GeV}^{2}$ \\
\hline & &
\end{tabular}

experiment from the simultaneous fit is found to $2.3 \sigma$. From Belle data, the asymptotic value $B$ obtained from fit(B) is $0.209 \pm 0.016 \mathrm{GeV}$ that is consistent with $\mathrm{pQCD}$ prediction of $\sim 0.185 \mathrm{GeV}$.

\section{References}

[1] J. L. Rosner, Phys. Rev. D 70, 034028 (2004).

[2] Z. Q. Liu et al., (Belle Collaboration), Phys. Rev. Lett. 108, 232001 (2012).

[3] M. Ablikim et al., (BES II Collaboration), Phys. Rev. Lett. 95, 262001 (2005); M. Ablikim et al., (BES III Collaboration), Phys. Rev. Lett. 106, 072002 (2011).

[4] K. Nakamura et al. (Particle Data Group), J. Phys. G 37, 075021 (2010).

[5] C.C. Zhang et al., (Belle Collaboration), Phys. Rev. D 86, 052002 (2012).

[6] B. Aubert et al., (BABAR Collaboration), Phys. Rev. D 80, 052002 (2009).

[7] B. J. Behrend et al. (CELLO collaboration), Z. Phys. C 49, 401 (1991); J. Gronberg et al., (CLEO Collaboration), Phys. Rev. D 5733 (1998).

[8] S.J. Brodsky, F.-G. Cao and G.F. de Téramond, Phys. Rev. D 84, 033001 (2011); Phys. Rev. D 84, 075012 (2011); and references therein.

[9] P. del Amo Sanchez et al., (BABAR Collaboration), Phys. Rev. D 84, 052001 (2011).

[10] S. Uehara et al., (Belle Collaboration), Phys. Rev. D 86, 092007 (2012).

[11] S. J. Brodsky, T. Kinoshita, and H. Terasawa, Phys. Rev. D 4, 1532 (1971). 